\title{
A METRIC FORM OF MICROTRANSITIVITY
}

\author{
AARNO HOHTI
}

\begin{abstract}
We prove that every homogeneous compact metrizable space $X$ has a compatible metric for which $X$ is Lipschitz homogeneous and for which the group $L(X)$ of Lipschitz homeomorphisms of $X$ acts Lipschitz microtransitively on $X$.
\end{abstract}

1. Introduction. This paper gives a metric analogue to what is called the "microtransitivity" of the action of the homeomorphism group of a compact metrizable homogeneous space. Homogeneous topological spaces have been studied by several authors after their definition by W. Sierpinski in [12]. A step forward was made possible in 1965 when Effros [2] proved a theorem concerning "smoothness" of the actions of Polish transformation groups. After its discovery in 1975 by Ungar [13] it has been used in several papers on homogeneous spaces written after 1975. As the work of Hagopian [3, 4], Ungar [13, 14], Jones [7], Rogers [11], Lewis [8, 9], Phelps [10] and Ancel [1] shows, many old problems have been solved and some old proofs have been simplified by the use of Effros's theorem in the form of microtransitivity.

For each compact metrizable space $X$, the group $H(X)$ of homeomorphisms of $X$ onto itself (equipped with the compact-open topology) is a Polish transformation group on $X$. It follows from Effros [2, Theorem 2.1], that if $G$ is a Polish transformation group acting transitively on a Polish space $X$, then for each $x \in X$ the map $T_{x}: G \rightarrow X$, given by $T_{x}(g)=g(x)$, is open. This implies that if $(X, d)$ is a homogeneous compact metric space, then $H(X)$ acts microtransitively on $X$ : For each $\delta>0$ there is an Effros number $\varepsilon(\delta)>0$ such that if $x, y \in X, d(x, y)<\varepsilon(\delta)$, then $f(x)=y$ for an $f \in H(X)$ satisfying $d(f(z), z)<\delta$ for all $z \in X$ (i.e., $f$ is a $\delta$-homeomorphism).

A Lipschitz homeomorphism is a surjective map $f: X \rightarrow Y$ of a metric space $(X, d)$ onto a metric space $(Y, \sigma)$ for which there is an $L \geqslant 1$ with

$$
L^{-1} d(x, y) \leqslant \sigma(f x, f y) \leqslant L d(x, y) \text { for all } x, y \in X .
$$

The least such $L$ is denoted by $\operatorname{bilip}(f)$. If $\operatorname{bilip}(f) \leqslant \lambda$, then $f$ is called a $\lambda$-Lipschitz homeomorphism. For each $\lambda \geqslant 1, L_{\lambda}(X, d)$ denotes the set of all $\lambda$-Lipschitz homeomorphisms of $(X, d)$ and we put $L(X, d)=\bigcup\left\{L_{\lambda}(X, d): \lambda \geqslant 1\right\}$. We call $(X, d)$ Lipschitz homogeneous [15] provided that for each pair $x, y$ of points of $X$ there is an $f \in L(X, d)$ such that $f(x)=y$. It has been shown in [6]

Received by the editors June 12, 1984 and, in revised form, April 2, 1985.

1980 Mathematics Subject Classification. Primary 54H15, 54E35.

Key' words and phrases. Homogenous, Lipschitz homeomorphism, homeomorphism group. 
that for every homogeneous compact metrizable space $X$ there is a compatible metric $d$ such that $(X, d)$ is Lipschitz homogeneous.

Let $(X, d)$ be a metric space. We say that the group $L(X, d)$ acts Lipschitz microtransitively on $(X, d)$ if for each $\delta>0$ and all $x \in X$ there is an $\varepsilon>0$ with the following property: If $y \in X$ and $d(x, y)<\varepsilon$, then there is a $\delta$-homeomorphism $f \in L(X, d)$ such that $f(x)=y$ and $\log (\operatorname{bilip}(f))<\delta$. In particular, if $X$ is compact, then $L(X, d)$ acts Lipschitz microtransitively on $(X, d)$ iff for each $\delta>0$ there is an $\varepsilon>0$ such that the $\delta$-homeomorphisms in $L_{1+\delta}(X, d)$ are " $\varepsilon$-transitive" on $(X, d)$.

Given a Lipschitz homogeneous space $(X, d)$, define for each pair $x, y$ of points of $X$ the number

$$
L_{x y}=\inf \{\operatorname{bilip}(f): f \in L(X, d), f(x)=y\} .
$$

Thus, if $L(X, d)$ acts Lipschitz microtransitively on $(X, d)$, then the formula $\sigma(x, y)=d(x, y)+\log \left(L_{x y}\right)$ defines a compatible "inner" metric on $X$. (The converse is true for compact $X$; see Proposition 3.3.) However, there are nice compact Lipschitz homogeneous spaces $(X, d)$ for which $L(X, d)$ does not act Lipschitz microtransitively on $(X, d)$. Equip the Hilbert cube $Q=[-1,1]^{N}$ with the metric $d$ given by

$$
d(x, y)=\max \left\{2^{-k}\left|x_{k}-y_{k}\right|: k \in N\right\} .
$$

It has been shown in [5] that $(Q, d)$ is Lipschitz homogeneous. On the other hand, it follows from [15, Theorem 3.4] that if $f:(Q, d) \rightarrow(Q, d)$ is any Lipschitz homeomorphism mapping the corner point $(1,1,1, \ldots)$ to the point $(0,0,0, \ldots)$, then necessarily $\operatorname{bilip}(f) \geqslant \sqrt{2}$. Virtually the same proof shows that for any Lipschitz homeomorphism $f \in L(X, d)$ mapping a point $(0,0,0, \ldots, 0,1,1,1, \ldots)$ to the point $(0,0,0, \ldots)$, one has $\operatorname{bilip}(f) \geqslant \sqrt{2}$. Thus, $L(Q, d)$ does not act Lipschitz microtransitively on $(Q, d)$. (However, there is an $L<\infty$ such that $L_{x y} \leqslant L$ for all $x, y \in Q$; see [5].) In this paper we prove that every homogeneous compact metrizable space $X$ has a compatible metric $d$ under which the action of $L(X, d)$ is Lipschitz microtransitive.

2. The main result. Our result will follow as a corollary to a sequence of five consecutive lemmas. In the sequel we assume that $(X, d)$ is a homogeneous compact metric space. We consider $H(X)$ with the complete metric $\tilde{d}$ given by

$$
\tilde{d}(f, g)=\sup \left\{d(f x, g x)+d\left(f^{-1} x, g^{-1} x\right): x \in X\right\} .
$$

Let $f \in H(X)$ and let $\varepsilon>0$. We define the modulus of uniform continuity of $f$ with respect to $\varepsilon$ by setting

$$
\omega_{\varepsilon}(f)=\sup \left\{\delta>0: d(x, y)<\delta \text { implies } d(f x, f y), d\left(f^{-1} x, f^{-1} y\right)<\varepsilon\right\} .
$$

Note that $\omega_{\varepsilon}$ is defined because each element of $H(X)$ is uniformly continuous. Hence, we obtain a map $\omega_{\varepsilon}: H(X) \rightarrow R$, where $R$ denotes the set real numbers.

2.1. Lemma. For each $\varepsilon>0$, the $\varepsilon$-modulus of continuity $\omega_{\varepsilon}: H(X) \rightarrow R$ is lower semicontinuous. 
Proof. Let $f \in H(X)$ and suppose that $\omega_{\varepsilon}(f)>t$. We must find a $\delta>0$ such that $\tilde{d}(f, g)<\delta$ implies $\omega_{\varepsilon}(g)>t$. Choose an $\alpha>0$ for which $\omega_{\varepsilon}(f)>t+\alpha$. Since $X$ is compact, there is a $\delta>0$ such that $d(x, y)<\omega_{\varepsilon}(f)-\alpha$ implies $d(f x, f y)$, $d\left(f^{-1} x, f^{-1} y\right)<\varepsilon-2 \delta$. Indeed, suppose that this does not hold. Then for each $n \in N$ we can find $x_{n}, y_{n} \in X$ such that $d\left(x_{n}, y_{n}\right)<\omega_{\varepsilon}(f)-\alpha$ but either $d\left(f x_{n}, f y_{n}\right) \geqslant \varepsilon-1 / n$ or $d\left(f^{-1} x_{n}, f^{-1} y_{n}\right) \geqslant \varepsilon-1 / n$. By passing to a subsequence if necessary, we can assume that $d\left(f x_{n}, f y_{n}\right) \geqslant \varepsilon-1 / n$ for all $n$.

Since $X$ is compact, $\left\langle x_{n}\right\rangle$ has a subsequence $\left\langle x_{n_{k}}\right\rangle$ converging to a point $x$. Similarly, $\left\langle y_{n_{k}}\right\rangle$ has a subsequence $\left\langle y_{n_{h(1)}}\right\rangle$ converging to a point $y$. We have $d(x, y) \leqslant \omega_{\varepsilon}(f)-\alpha<\omega_{\varepsilon}(f)$, but $d(f x, f y) \geqslant \varepsilon$, which contradicts the definition of $\omega_{\boldsymbol{\varepsilon}}$.

To finish the proof, note that $d(x, y)<\omega_{\varepsilon}(f)-\alpha$ implies

$$
d(g x, g y) \leqslant d(f x, g x)+d(f x, f y)+d(f y, g y)<\varepsilon
$$

whenever $\tilde{d}(f, g)<\delta$, and similarly for $d\left(g^{-1} x, g^{-1} y\right)$. Thus, $\tilde{d}(f, g)<\delta$ implies $\omega_{f}(g)>\omega_{\varepsilon}(f)-\alpha>t$.

Let $\delta>0$. A subset $F$ of $H(X)$ is called $\delta$-transitive if $d(x, y) \leqslant \delta$ implies that there is an $f \in F$ mapping $x$ to $y$. Now let $\varepsilon>0$ and suppose that $F$ is $\delta$-transitive. We put $X_{\delta}=\{(x, y) \in X \times X: d(x, y) \leqslant \delta\}$ and define a map $\varphi_{\varepsilon}^{F}: X_{\delta} \rightarrow R$ by

$$
\varphi_{\varepsilon}^{F}(x, y)=\sup \left\{\omega_{\varepsilon}(f): f \in F, f(x)=y\right\} .
$$

2.2. LemMA. Let $\delta>0$ and let $F$ be an open $\delta$-transitive subset of $H(X)$. Then for each $\varepsilon>0$ the map $\varphi_{\varepsilon}^{F}$ is lower semicontinuous.

Proof. The claim follows from the lower semicontinuity of $\omega_{\varepsilon}$ and Effros's theorem. To be precise, let $(x, y) \in X_{\delta}$ and let $\varphi_{\varepsilon}^{F}(x, y)>t$. We must find a neighbourhood $U$ of $(x, y)$ in $X_{\delta}$ such that $\varphi_{\varepsilon}^{F}(z, w)>t$ for all $(z, w) \in U$. It follows from the definition of $\varphi_{\varepsilon}^{F}$ that there is an $f \in F$ with $\omega_{\varepsilon}(f)>t$ and $f(x)=y$. By $2.1 \omega_{f}$ is lower semicontinous, and hence we can find a neighbourhood $V$ of $f$ in $F$ such that $g \in V$ implies $\omega_{\varepsilon}(g)>t$. Since $F$ is open, we can choose a neighbourhood $W$ of id in $H(X)$ satisfying the condition $g f h^{-1} \in V$ for all $g, h \in W$. By Effros's theorem $U=\left(T_{x}[W] \times T_{y}[W]\right) \cap X_{\delta}$ is a neighbourhood of $(x, y)$ in $X_{\delta}$. Now for all $(z, w) \in U$ there exist $g, h \in W$ with $g(x)=z$ and $h(y)=w$. Then $\left(h f g^{-1}\right)(z)$ $=w$ and $\omega_{\varepsilon}\left(h f g^{-1}\right)>t$. Consequently, $\varphi_{\varepsilon}(z, w)>t$ for all $(z, w) \in U$.

2.3. Lemma. For each $\delta>0$ and each $\delta$-transitive neighbourhood $U$ of id in $H(X)$, there is a compact $\delta$-transitive subset $K$ of $H(X)$ such that id $\in K \subset U$.

Proof. We can assume that $U$ is open. Since $X_{\delta}$ is compact and $\varphi_{2-1}^{U}$ is lower semicontinous by 2.2 , we have

$$
r_{1}=\frac{1}{2} \inf \left\{\varphi_{2^{-1}}^{U}(x, y):(x, y) \in X_{\delta}\right\}>0 .
$$

Let $U_{1}=\left\{f \in U: \omega_{2^{-1}}(f)>r_{1}\right\}$. Since $\omega_{2^{-1}}$ is lower semicontinuous, $U_{1}$ is open, and certainly id $\in U_{1}$ because $\omega_{\alpha}(g) \leqslant \omega_{\alpha}(\mathrm{id})$ for all $\alpha>0$ and $g \in H(X)$. It immediately follows from the definition of $U_{1}$ that $U_{1}$ is $\delta$-transitive.

We proceed inductively and assume that $U_{n}$ is defined. Let

$$
r_{n+1}=\frac{1}{2} \inf \left\{\varphi_{2^{n-1}}^{U_{n-1}}(x, y):(x, y) \in X_{\delta}\right\}>0,
$$


and put $U_{n+1}=\left\{f \in U_{n}: \omega_{2-n-1}(f)>r_{n+1}\right\}$. In this way we obtain a sequence $\left\langle U_{n}\right\rangle$ of open $\delta$-transitive neighbourhoods of id in $H(X)$.

Given $(x, y) \in X_{\delta}$, there is, for each $n \in N$, an $f_{n} \in U_{n}$ with $f(x)=y$. By construction the sequence $\left\langle f_{n}\right\rangle$ is equicontinuous. (Indeed, let $\varepsilon>0$. Choose an $m \in N$ for which $2^{-m}<\varepsilon$. Then $d(z, w)<r_{m}$ implies $d\left(f_{k} z, f_{k} w\right)<\varepsilon$ for all $k \geqslant m$. For each $i \in\{1, \ldots, m-1\}$ choose an $\alpha_{i}>0$ such that $d(z, w)<\alpha_{i}$ implies $d\left(f_{i} z, f_{i} w\right)<\varepsilon$. Let $\alpha=\min \left\{r_{m}, \alpha_{1}, \ldots, \alpha_{m-1}\right\}$. Then $\alpha$ witnesses the equicontinuity of $\left\langle f_{n}\right\rangle$ with respect to $\varepsilon$.) By Ascoli's theorem the sequence $\left\langle f_{n}\right\rangle$ is relatively compact as a subset of $H(X)$, and thus there exist a subsequence $\left\langle f_{n_{k}}\right\rangle$ and an $f \in H(X)$ such that $f_{n_{k}} \rightarrow f$. Clearly $f(x)=y$. Therefore, if we let $K$ consist of all limits of sequences $\left\langle f_{n}\right\rangle$, where $f \in V_{n}$, then $K$ is a $\delta$-transitive closed subset of $H(X)$. Since $\omega_{\alpha}(g) \leqslant \omega_{\alpha}\left(\right.$ id) for all $\alpha>0$ and $g \in H(X)$, we have id $\in \bigcap\left\{V_{n}\right.$ : $n \in N\} \subset K$. Finally, $K$ is equicontinuous. In fact, let $s>0$, let $f \in K$ and let $\left\langle f_{n}\right\rangle$ be a sequence such that $f_{n} \in V_{n}$ and $f_{n} \rightarrow f$. Choose an $m \in N$ with $2^{-m}<\varepsilon / 3$. There is an $n \geqslant m$ such that $\tilde{d}\left(f_{n}, f\right)<\varepsilon / 3$. But $d(x, y)<r_{m}$ implies

$$
d(f x, f y) \leqslant d\left(f x, f_{n} x\right)+d\left(f_{n} x, f_{n} y\right)+d\left(f_{n} y, f y\right)<\varepsilon,
$$

and thus $r_{m}$ witnesses the equicontinuity of $K$ with respect to $\varepsilon$.

For each finite sequence $i_{1}, \ldots, i_{n}$ of elements of $N$, let

$$
a\left(i_{1}, \ldots, i_{n}\right)=\left(1+2^{-i_{1}}\right)^{-1} \ldots\left(1+2^{-i_{n}}\right)^{-1} .
$$

We assume that the metric $d$ on $X$ is bounded above by 1 . For each $n>0$, let $p_{n}$ be a positive integer such that $\left(1+2^{-n}\right)^{p_{n}}<1 / n$. The proof of the following lemma is due to the referee and shortens the original proof given by the author.

2.4. LemMA. There is a sequence $\left\langle K_{n}\right\rangle$ of compact symmetric subsets of $H(X)$ such that each $K_{i}$ contains the identity map and

(1) $K_{i}$ is $\delta_{i}$-transitive for some positive $\delta_{i}$,

(2) for each $\varepsilon>0$, the union of all $K_{i_{1}} \cdots K_{i_{n}}$ such that $a\left(i_{1}, \ldots, i_{n}\right) \geqslant \varepsilon$ is equicontinuous.

Proof. For each $r>0$, let $G(r)=\{f \in H(X): \tilde{d}(f$, id $) \leqslant r\}$. By induction we construct sequences $\left\langle r_{n}\right\rangle$ and $\left\langle\lambda_{n}\right\rangle$ of positive real numbers and a sequence $\left\langle K_{n}\right\rangle$ of compact symmetric subsets of $H(X)$ with the following properties.

(a) $2 p_{n} r_{n} \leqslant \min \left\{p_{n-1} r_{n-1}, \lambda_{n-1}, 1 / n\right\}$.

(b) id $\in K_{n} \subset G\left(r_{n}\right)$, and $K_{n}$ is $\delta_{n}$-transitive for some $\delta_{n}>0$.

(c) If $f_{i} \in K_{1} \cup \cdots \cup K_{n}$ for $1 \leqslant i \leqslant p_{n}, g_{i} \in G\left(\lambda_{n}\right)$ for $1 \leqslant i \leqslant p_{n}+1$, and $h=g_{1} \circ f_{1} \circ \cdots \circ g_{p_{n}} \circ f_{p_{n}} \circ g_{p_{n}+1}$, then $d(x, y) \leqslant \lambda_{n}$ implies $d(h(x), h(y)) \leqslant 1 / n$ for all $x, y \in X$.

To begin, set $r_{1}=1$ and use Lemma 2.3 to obtain $K_{1}$ satisfying (b). Set $\lambda_{1}=1$. Since $d$ is bounded above by 1 , condition (c) is satisfied.

Now let $n>1$ and inductively assume that we already have defined $r_{i}, K_{i}$ and $\lambda_{i}$ for $1 \leqslant i \leqslant n-1$. First choose $r_{n}>0$ to satisfy (a). Next use Lemma 2.3 to choose $K_{n}$ that satisfies (b). We select $\lambda_{n}$ as follows. The equicontinuity of $K_{1} \cup \cdots \cup K_{n}$ 
allows us to choose a decreasing sequence $\mu_{0}, \mu_{1}, \ldots, \mu_{p_{n}}$ of positive real numbers with the following properties:

(d) $\mu_{0}=1 / n$.

(e) For $1 \leqslant i \leqslant p_{n}$ and for each $f \in K_{1} \cup \cdots \cup K_{n}, d(x, y) \leqslant \mu_{1}$ implies $d(f(x), f(y)) \leqslant \mu_{i-1} / 3$ whenever $x, y \in X$.

Now set $\lambda_{n}=\mu_{p_{n}} / 3$. It is easily seen that (c) is valid for this choice of $\lambda_{n}$. This completes the inductive step and shows that the sequences $\left\langle r_{n}\right\rangle,\left\langle K_{n}\right\rangle$ and $\left\langle\lambda_{n}\right\rangle$ can be constructed as desired. Notice that (a) implies $r_{n} \leqslant 1 / n$; hence the claim that $K_{n} \subset G(1 / n)$ follows from (b).

For each $n>0$, set $L_{n}=\bigcup\left\{K_{i_{1}} \cdots K_{i_{r}}: a\left(i_{1}, \ldots, i_{r}\right) \geqslant 1 / n\right\}$. We prove the second contention of 2.4 by first establishing the weaker result that for every $h \in L_{n}, d(x, y) \leqslant \lambda_{n}$ implies $d(h(x), h(y)) \leqslant 1 / n$ for all $x, y \in X$.

Thus, let $h \in L_{n}$. Then $h \in K_{i_{1}} \cdots K_{i_{r}}$ where $a\left(i_{1}, \ldots, i_{r}\right) \geqslant 1 / n$. Since $(1+$ $\left.2^{-i}\right) \leqslant\left(1+2^{-n}\right) \leqslant(1 / n)^{1 / p_{n}}$ for $1 \leqslant i \leqslant n$, there are at most $p_{n}$ indices $i_{j}$ for which $i_{j} \leqslant n$. Therefore $h$ decomposes as $h=g_{1} \circ f_{1} \circ \cdots \circ g_{p_{n}} \circ f_{p_{n}} \circ g_{p_{n}+1}$, where $f_{k} \in K_{1} \cup \cdots \cup K_{n}$ for $1 \leqslant k \leqslant p_{n}$ and each $g_{k}$ is the composition of a set of elements from $K_{n+1} \cup K_{n+2} \cup \cdots$ for $1 \leqslant k \leqslant p_{n}+1$. For each $i>n$ the condition $\left(1+2^{-i}\right)^{p_{i}}<1 / i<1 / n$ implies that there are at most $p_{i}$ numbers $\left(1+2^{-i},\right)$ $(1 \leqslant j \leqslant r)$ for which $i_{j}=i$. Hence, for each $i>n$, each $g_{k}$ has at most $p_{i}$ factors from $K_{i}$. Also $K_{i} \subset G\left(r_{i}\right)$ for $i>n$. Since $\tilde{d}\left(g_{k}\right.$, id $) \leqslant$ the sum of the distance from the factors of $g_{k}$ to id, we have

$$
\tilde{d}\left(g_{k}, \mathrm{id}\right)<\sum_{i=n+1}^{\infty} p_{i} r_{i} \text { for } 1 \leqslant k \leqslant p_{n}+1 .
$$

Condition (a) implies that

$$
\sum_{i=n+1}^{\infty} p_{i} r_{i} \leqslant 2 p_{n+1} r_{n+1} \leqslant \lambda_{n} .
$$

Thus $1 \leqslant k \leqslant p_{n}+1$ implies $g_{k} \in G\left(\lambda_{n}\right)$. Now our assertion follows from condition (c).

To conclude the proof, let $\varepsilon>0$ and set $L=\bigcup\left\{K_{i_{1}} \cdots K_{i_{r}}: a\left(i_{1}, \ldots, i_{r}\right) \geqslant \varepsilon\right\}$. We must prove that $L$ is equicontinuous. Let $\mu>0$. Choose $n \geqslant 1$ so that $1 / n<\min \{\varepsilon, \mu\}$. Then $L \subset L_{n}$. Let $h \in L_{n}$. The assertion proved above implies that if $x, y \in X$ and $d(x, y) \leqslant \lambda_{n}$, then $d(h(x), h(y)) \leqslant 1 / n \leqslant \mu$. We conclude that $L$ is equicontinuous.

2.5. Lemma. Let $\left\langle K_{n}\right\rangle$ be as in 2.4. Then there is a compatible metric $\sigma$ of $X$ such that the elements of $K_{n}$ are $\left(1+2^{-n}\right)$-Lipschitz homeomorphisms in $(X, \sigma)$ for all $n \in N$.

Proof. For each finite sequence $i_{1}, \ldots, i_{n}$ of positive integers, we define a pseudometric $\sigma_{i_{1}} \ldots i_{n}$ by the formula

$$
\sigma_{i_{1}} \cdots i_{n}(x, y)=\sup \left\{d(f x, f y): f \in K_{i_{1}} \cdots K_{i_{n}}\right\} .
$$


Since $K_{i_{1}} \cdots K_{i_{n}}$ is compact and thus equicontinuous, $\sigma_{i_{1}} \cdots i_{n}$ is a continuous pseudometric on $X$. On the other hand, id $\in K_{i_{1}} \cdots K_{i_{n}}$, and, hence, in fact, $\sigma_{i_{1}} \cdots i_{n}$ is a metric. Let

$$
\sigma(x, y)=\sup \left\{a\left(i_{1}, \ldots, i_{n}\right) \sigma_{i_{1} \cdots i_{n}}(x, y): i_{1}, \ldots, i_{n}, n \in N\right\} .
$$

Obviously $\sigma$ is a metric of $X$, stronger than $d$. To show that $\sigma$ is compatible with the topology of $X$, we must prove that for each $\varepsilon>0$ there is a $\delta>0$ such that $d(x, y)<\delta$ implies $\sigma(x, y)<\varepsilon$. By 2.4 there is a $\delta>0$ such that if $a\left(i_{1}, \ldots, i_{n}\right) \geqslant$ $\varepsilon / \operatorname{diam}(X)$ and $f \in K_{i_{1}} \cdots K_{i_{n}}$, then $d(x, y)<\delta$ implies $d(f x, f y)<\varepsilon$. Thus, $d(x, y)<\delta$ implies $a\left(i_{1}, \ldots, i_{n}\right) \sigma_{i_{1} \ldots i_{n}}(x, y)<\varepsilon$ for all $i_{1}, \ldots, i_{n} \in N$, as desired.

Let $f \in K_{n}$, let $x, y \in X$ and let $\alpha>0$. Choose $i_{1}, \ldots, i_{m} \in N$ such that

$$
\sigma(f x, f y) \leqslant a\left(i_{1}, \ldots, i_{m}\right) \sigma_{i_{1} \ldots i_{m}}(f x, f y)+\alpha .
$$

We have

$$
\begin{aligned}
\sigma_{i_{1} \cdots i_{m}}(f x, f y) & =\sup \left\{d(g f x, g f y): g \in K_{i_{1}} \cdots K_{i_{m}}\right\} \\
& \leqslant \sup \left\{d(h x, h y): h \in K_{i_{1}} \cdots K_{i_{m}} K_{n}\right\} .
\end{aligned}
$$

Thus,

$$
\begin{aligned}
\sigma(f x, f y) & \leqslant \frac{a\left(i_{1}, \ldots, i_{m}\right)}{a\left(i_{1}, \ldots, i_{m}, n\right)}\left(a\left(i_{1}, \ldots, i_{m}, n\right) \sigma_{i_{1} \cdots i_{m}, n}(x, y)\right)+\alpha \\
& \leqslant\left(1+2^{-n}\right) \sigma(x, y)+\alpha .
\end{aligned}
$$

Since $K_{n}$ is symmetric (i.e, $K_{n}=K_{n}^{-1}$; this follows from the fact that $\omega_{\alpha}(g)=$ $\omega_{\alpha}\left(g^{-1}\right)$ for all $\left.g \in H(X)\right)$, we infer that each element of $K_{n}$ is a $\left(1+2^{-n}\right)$-Lipschitz homoemorphism of $(X, \sigma)$.

2.6. THEOREM. For each homogeneous compact metrizable space $X$ there is a compatible metric $\sigma$ such that $L(X, \sigma)$ acts Lipschitz microtransitively on $(X, \sigma)$.

Proof. Let $\left\langle K_{n}\right\rangle$ be the sequence promised by 2.4 . Note that the proof of 2.4 shows that we can assume $K_{n} \subset G_{n}$, where $\left\langle G_{n}\right\rangle$ is a neighbourhood base of id in $H(X)$. Let $\sigma$ be the metric given in 2.5. For every $\varepsilon>0$ we can find an $n$ such that the elements of $K_{n}$ are $\varepsilon$-homeomorphisms of $(X, \sigma)$ and $2^{-n}<\varepsilon$. Since $X$ is compact and $\sigma$ is compatible with the topology of $X, K_{n}$ is $\delta$-transitive for some $\delta>0$. Thus, $\sigma(x, y)<\delta$ implies that there is an $\varepsilon$-homemorphism $f \in K_{n}$ such that $f(x)=y$ and $\operatorname{bilip}(f) \leqslant 1+2^{-n}<1+\varepsilon$.

3. Topological microtransitivity. The reader might wonder whether the first half of Lipschitz microtransitivity, the topological microtransitivity of the group of Lipschitz homeomorphisms, is true for all compact Lipschitz homogeneous spaces. The answer is positive even though Effros's theorem cannot be applied, since, in general, $L(X, d)$ is not Polish for a compact (Lipschitz homogeneous) metric space $(X, d)$.

3.1. THEOREM. Let $(X, d)$ be a Lipschitz homogeneous compact metric space. Then for each $x \in X$, the map $T_{x}: L(X, d) \rightarrow X$ is open. 
Proof. Let $U$ be a nonempty open subset of $L(X, d)$. To show that $T_{x}[U]$ is open, let $y \in T_{x}[U]$ with $f(x)=y, f \in U$. Choose a symmetric neighbourhood $V$ of id such that $\left(\bar{V}^{2}\right) f \subset U$. Since $L(X, d)$ is separable and metrizable, there exists a sequence $\left\langle f_{n}\right\rangle$ of elements of $L(X, d)$ such that $L(X, d)=\bigcup\left\{f_{n} V: n \in N\right\}$. Since $(X, d)$ is Lipschitz homogeneous, we have $T_{x}[L(X, d)]=X$ and thus

$$
X=\bigcup\left\{T_{y}\left[f_{n} V\right]: n \in N\right\}=\bigcup\left\{f_{n}\left[T_{y}[V]\right]: n \in N\right\} .
$$

Now $X$ is of the second category in itself, and hence one of the sets $f_{n}\left[T_{y}[V]\right]$ is of the second category in $X$. Since the maps $f_{n}$ are homeomorphisms, it follows that $T_{y}[V]$ is of the second category in $X$. Now $\bar{V}=\bigcup\left\{\bar{V} \cap L_{n}(X, d): n \in N\right\}$. By Ascoli's theorem and the compactness of $X$ each $L_{n}(X, d)$ is a compact subset of $L(X, d)$, and hence $T_{y}[\bar{V}]$ is $\sigma$-compact. There is $m$ such that $T_{y}\left[\bar{V} \cap L_{m}(X, d)\right]$ is a (closed) second category subset of $X$, and thus int ${ }_{X} T_{y}[\bar{V}] \neq \varnothing$. Choose a $g \in \bar{V}$ for which $g(y) \in$ int $_{X} T_{y}[\bar{V}]$. Then $W=g^{-1}\left[\right.$ int $\left._{X} T_{y}[\bar{V}]\right]$ is an open neighbourhood of $y$ and $y \in W \subset T_{y}\left[\bar{V}^{2}\right]=T_{x}\left[\bar{V}^{2} f\right] \subset T_{x}[U]$, which shows that $T_{x}[U]$ is open.

Given an $x \in X$, denote by $L_{x}(X, d)$ the stabilizer of $x$ in $L(X, d)$; i.e.,

$$
L_{x}(X, d)=\{f \in L(X, d): f(x)=x\} .
$$

The natural map $L(X, d) \rightarrow L(X, d) / L_{x}(X, d)$ is open, and if $(X, d)$ is compact and Lipschitz homogeneous, then by 3.1 the map $F_{x}: L(X, d) / L_{x}(X, d) \rightarrow X$ given by $F_{x}\left(g L_{x}(X, d)\right)=g(x)$ is open. Therefore, we obtain the following corollary (see [13, Theorem 3.2]).

3.2. Corollary. Let $(X, d)$ be a Lipschitz homogeneous compact metric space and let $x \in X$. Then $X$ is a quotient of $L(X, d)$ by $L_{x}(X, d)$ :

$$
L(X, d) / L_{x}(X, d) \cong X .
$$

Now suppose that $(X, d)$ is a compact metric space and that for every $r>0$ there is a $\delta_{r}>0$ such that $L_{1+r}(X, d)$ acts $\delta_{r}$-transitively on $(X, d)$. As in the proof of 3.1 , one can show that for each neighbourhood $U$ of id in $L(X, d)$ and for every $x \in X, T_{x}\left[L_{1+r}(X, d) \cap U\right]$ is a neighbourhood of $x$ in $X$. This yields a proof of the following

3.3. Proposition. Let $(X, d)$ be a compact metric space. Then $L(X, d)$ acts Lipschitz microtransitively on $(X, d)$ iff the formula $\sigma(x, y)=d(x, y)+\log L_{x y}$ defines a compatible metric of $X$.

\section{REFERENCES}

1. F. D. Ancel, A proof and some applications of a theorem of E. G. Effros (preprint).

2. E. G. Effros, Transformation groups and $C^{*}$-algebras, Ann. of Math. (2) 81 (1965), 38-55.

3. C. L. Hagopian, Homogeneous plane continua, Houston J. Math. 1 (1975), 35-44.

4. Indecomposable homogeneous plane continua are hereditarily indecomposable, Trans. Amer. Math. Soc. 224 (1976), 339-350.

5. A. Hohti, On Lipschitz homogeneity of the Hilbert cube, Trans. Amer. Math. Soc. 291 (1985), $75-86$.

6. A. Hohti and H. Junnila, A note on homogeneous metrizable spaces, Houston J. Math. (to appear).

7. F. B. Jones, Use of a new technique in homogeneous continua, Houston J. Math. 1 (1975), 57-61.

8. W. Lewis, Almost chainable homogeneous continua are chainable, Houston J. Math. 7 (1981), $373-377$. 
9. Homogeneous tree-like continua, Proc. Amer. Math. Soc. 82 (1981), 470-472.

10. J. K. Phelps, Homogeneity and groups of homeomorphisms, Topology Proc. 6 (1981), 371-404.

11. J. T. Rogers, Jr., Decompositions of homogeneous continua, Pacific J. Math. 99 (1982), 137-144.

12. W. Sierpinski, Sur une propriété topologique des ensembles dénombrables denses en soi, Fund. Math. 1 (1920), 11-16.

13. G. S. Ungar, On all kinds of homogeneous spaces, Trans. Amer. Math. Soc. 121 (1975), 393-400.

14. Countable dense homogeneity and n-homogeneity, Fund. Math. 99 (1978), 155-160.

15. J. Väisälä, Lipschitz homeomorphisms of the Hilbert cube, Topology Appl. 11 (1980), 103-110.

Department of Mathematics, University of Helsinki, Hallituskatu 15, SF - 00100 Helsinki 10 , FINI.AND 